\title{
Hiperplasia hipofisiaria sugerente de macroadenoma en un caso de hipotiroidismo primario
}

\author{
Pamela Pérez O. ${ }^{\text {; }}$ Anjbal Espinoza G. ${ }^{\text {; }}$ Luisa Kemm V. '; Fernando Cassorla G.'
}

\begin{abstract}
Resumen
En una niña con hipatioidismo primario debicia a tiroides sublingual y ralla baja desde los dos años de edad, la resonancio magnético mostró aunento del ramaño de la hipótisis sugerente de macroadenoma, que después de una prueba terapéulico con hormona tiroidea recuperó el aspecto normal. El hiposiroidismo primorio crónico puede producir aumento de volumen hipofisiario indishinguible del cousado por un macroadenomo en los exórrenes por imágenes. El tratomiento con hormono tiroidea permile el diagnóstico diferencial entre ambas entidades.
\end{abstract}

[Palabras clave: hipoliroidismo primario, pseudo macroadenomo hipofisisario, talla baja.]

\section{Primary hipothyroidism simulating pituitary macroadenoma}

In a girl with primary hypathyroidism coused by a lingual thyrord gland and short slature starling ol age 2 years, mogneric resonance showed pituitary enlargement suggestive of macroadenoma, bul after a therapeutic lrici with thyroid hormone pituitary size returned to nomal. Long-siandirg primary hypothyroidism may produce images suggesting pituifary entargement and macroodenomo. The theropeutic trial with thyroid hormone can be useful in the differential diagnosis among both conditions.

[Key words: hipcthyroidism, pitultary neoplosm, short stature.]

El hipotiroidismo primario es frecuente en pediatría. Diversas alteraciones orgánicas o funcionales de la glándula tiroides pueden afectar la secreción de estas hormonas'. El hipotiroidismo secundario o terciario, por falla hipofisiaria - hipotalámica, es de frecuencia considerablemente menor ${ }^{2}$.

La edad de presentación del hipotiroidismo varía según la etiología y la intensidad de la disfunción tiroidea. Alteraciones en la embriogénesis de la glándula tiroides se manifiestan generalmente desde el recién nacido, causando hipotiroidismo congénito, que puede tener severas repercusiones sobre el crecimiento y desarrollo si no es tratado adecuadamente $e^{3}$.

El hipotiroidismo adquirido, por otro lado, se manifiesta generalmente durante la niñez o ado-

I. Universidad de Chile, Facultad de Medicina, Campus Centro. Insiicuto de Investigaciones Matemo-Infantil (1DIMI), Hospilal Clínico San Borja-Arriarán. lescencia. Su principal causa, después de establecerse la yodación de la sal en nuestro medio, es la tiroiditis autoinmune linfocitaria!.

Si el hipotiroidismo primario, congénito o adquirido, no es tratado adecuadamente, la pérdida de la retroalimentacion negativa de las hormonas troideas sobre la hipófisis produce crecimientos de magnitud variable de estas ${ }^{4-8}$, que a su vez pueden hacer necesario el diagnóstico diferencial con otros procesos expansivos hipofisiarios.

Se describe una nin̆a de cinco años con hipotiroidismo primario debido a un tiroides ectopico y signos de hiperplasia hipofisiaria sugerentes de macroadenona, que se resolvió con tratamiento con hormona tiroidea. Este caso enfatiza la necesidad de ser prudente en la interpretación del estudio pituitario por imágenes en caso de hipotiroidismo primario, y reafïma la necesidad de tratamiento médico del trastorno antes de considerar su derivación al neurocirujano. 


\section{Caso clínico}

Niña, procedente de otra ciudad, consultó por talla baja a los cinco años seis meses de edad. Embarazo y parto normales. Peso de nacimiento: $4040 \mathrm{~g}$. talla de nacimiento $52,5 \mathrm{~cm}$. Padre y madre sanos, tallas de 176 y $164 \mathrm{~cm}$ respectivamente. Sin antecedentes tnótbidos de importancia personales ri familiares. Su crecimiento lineal fue adecuado hasta los dos afios de edad, cuando su velocidad se redujo, Jlegando la talla a ser menor a $2 \mathrm{DE}$ a los 5 años de edad. Su desarrollo psicomotor fue normal. pero mostraba notoria pasividad y disminución de la actividad física, sin cefalea, galactorrea o alteraciones visuales. A los 5 años I0 Ineses de edad era una nî̉a tranquila, talla $105,4 \mathrm{~cm}$ (percentil 5), peso $23,3 \mathrm{~kg}$ (percentil 90), frecuencja cardíaca $60 x$ min, piel seca. No tenía bocio y no había desarrollo puberal. La edad ósea correspondía a dos años. La radiografía de silla turca mostró aumento significativo del volumen hipofisiario y en ta tomografia axial de hipófisis se registró un proceso expansivo intra y supraselar. En la resonancia magnética de silla turca se confirmo la masa intraselar y supraselar, cuyo diámetro aproximado era 17 mm en el sentido cefalo-caudal (figura la). La intensidad espontánea en TI de la rxurohipófisis estaba conservada y el medio de contraste produjo reforzamiento homogéneo de la masa. to que fue interprecado como sugerente de macroadenoma hipolisiario. La concentración de prolactina cra $82 \mathrm{ng} / \mathrm{ml}, \mathrm{T} 3: 59,7 \mathrm{ng} / \mathrm{dl} ; \mathrm{T} 4: 2,1 \mathrm{ug} / \mathrm{dl}$; T4 libre: 0,4 ug/dl y TSH: $85,7 \mathrm{mLL} / \mathrm{L}$. La cintigrafia tiroidea con $T c 99$ mostró ausencia de tejido tiroideo funcionante en la ubicación habitual de la glándula, pero se encontró un sitio de captación del trazador en la base de la lengua, compatible con tiroides sublingual (figura 2).

Dos meses después de iniciar tratamiento de reemplazo con levotiroxina 100 ug por día, una nueva resonancia nagnética de conirol reveló que la silla turca era de aspecto normal y estaba ocupada por una glándula hipofisiaria cuya configuración, tamaño y caracteriscicas eran también normales (figura Ib). No se encontró evidencia de lesión expansiva.

La niña estr actualmente en tratamiento de reemplazo con levotiroxina, eutiroidea clffica y bioquímicamente, con importante mejocía en su velocidad de crecimiento. Su T4. TSH y prolactina son normales (T4 libre $=1,8 \mathrm{ug} / \mathrm{dl}$, $\mathrm{TSH}=0,3 \mathrm{mUL} / \mathrm{L}, \mathrm{PRL}=8 \mathrm{ng} / \mathrm{ml}$ ).

\section{Comentario}

El hipotiroidismo primario varía en la forma de presentación dependiendo de su etiología y de la edad del paciente ${ }^{l}$. El (iroides ectópico y las dishormonogénesis pueden evolucionar mucho tiempo con síntomas leves o poco especifi$\cos ^{1}$, como en esta paciente cuya velocidad de crecimiento se deterioró varios años antes de consultar.

El hipotiroidismo primario puede inducir crecimiento hipofisiario por hiperplasia de las células tirotrofas y lactotrofas en respuesta a dismi- nucion de las hormonas tiroideas circulantes ${ }^{5.9}$. Ya en 1851 Riepce describió aumento de volumen de la hipófisis en un paciente con hipotiroidismo congénito ${ }^{10}$. La hiperplasia de Jos tirotropos aumenta la secreción de TSH para compensar la falla tiroidea. También pueden ocurrir incrementos de la secreción de otras hormonas hipofisiarias ${ }^{9}{ }^{10}$, como prolactina ${ }^{3-11}$ y gonadotrofinas ${ }^{10 .}{ }^{12}$, que pueden producir pubertad precoz. El tratamiento con hormona tiroidea

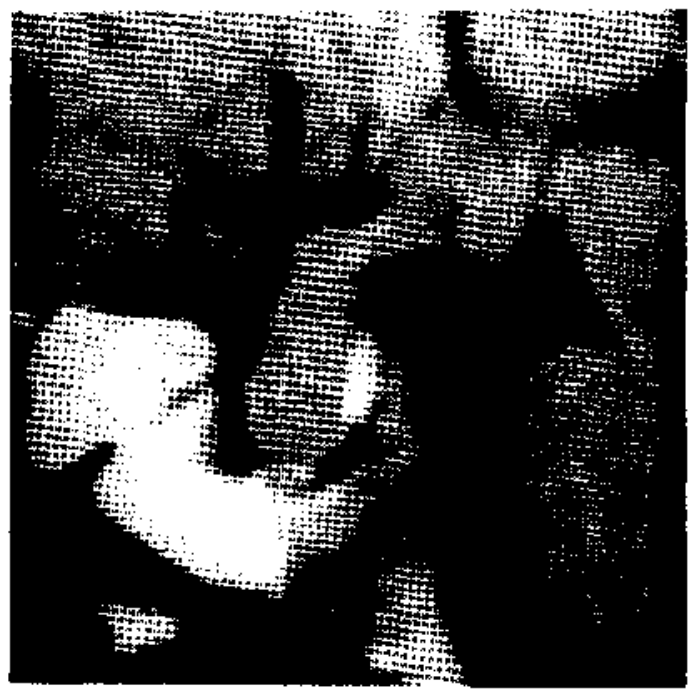

Figura 1a: Responancia nuclear magnćtica realizada antes del tratamiento. Se observa proceso expansivo intra y supraselar.

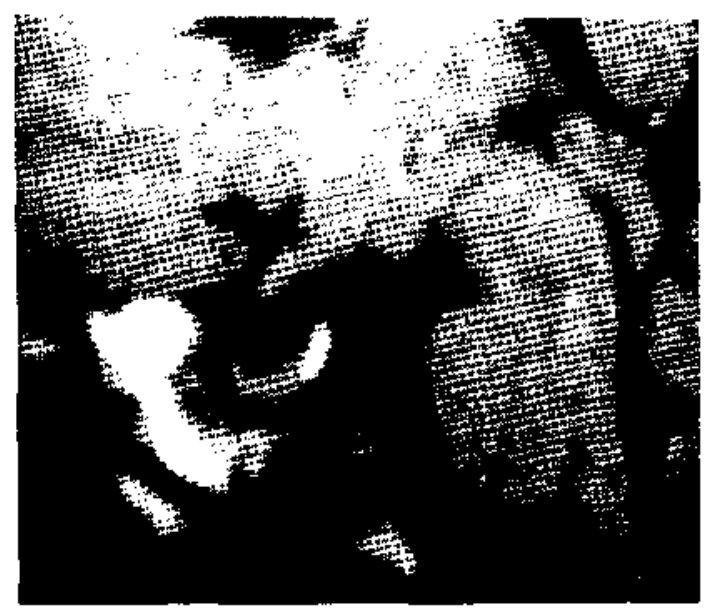

Figura 1b: Resonancia nuclear magnética realizada des. pués del tratamiento. Se observa resolución de la lesión expansiva. 


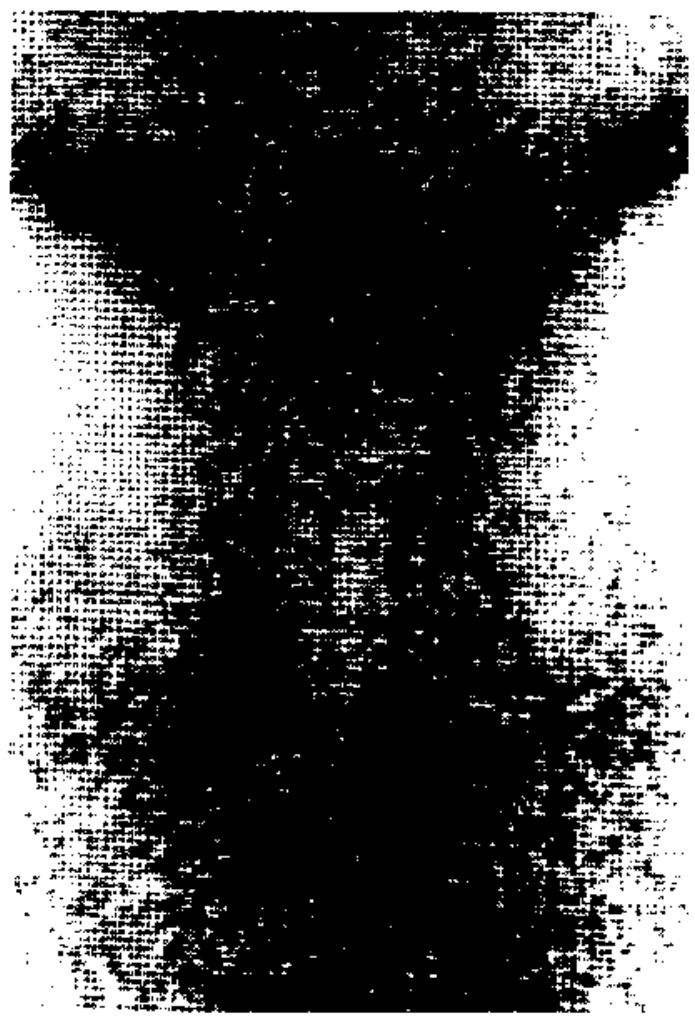

Figura 2: Cintigrafía tiroidea Te99. Se observa captación del radiofármaco a nivel de la base de la lengua.

induce la regresión total de este crecimiento hipofisiario ${ }^{5-8,11,12}$.

No está claro si, en humanos, esta biperplasia de los tirotropos podría evolucionar hacia un adenoma autónomo, como se ha demostrado en animales $4,5,11,13,14$. Sin embargo, en autopsias de 64 sujetos hipotiroideos no tratados se encontraron 12 casos de adenoma hipofisiario". Clínicamente esta hiperplasia bipofisiaria por hipotiroidismo primario puede manifestarse con signos neurologicos como cefalea o alteraciones visuales ${ }^{6,13,15}$; también corno galactorrea, sugiriendo prolactinoma ${ }^{17}$, o ser totalmente asintomática'. En nuestra paciente no hubo signos y síntomas neurológicos y el crecimiento hipofisiario se detect 6 como hallazgo de los exámenes por imágenes solicitados para estudio de su talla baja. Frente a un cuadro de esta naturaleza, el diagnóstico diferencial debe inclujr un adenoma productor de TSH o una resistencia central a hormonas tiroideas $5,16,18$, que pueden cursar con crecimiento hipofisiario y aumento de la
TSH. Sin embargo, estos cuadros están asociados con niveles circulantes elevados de hormonas tiroideas.

El hipotiroidismo clínico con confirmación bioquímica y la alteración estructural del tiroides sugirieron en nuestra paciente que se trataba de un hipotiroidismo primario con hiperplasia hipofisiaria. No obstante, la posibilidad de que la hiperplasia hipofisiaria causada por el hipotiroidismo primario pueda eventualmente derivar hacia el desarrollo de un adenoma, o de otro tumor hipofisiario no relacionado con el hipotiroidismo $0^{4,5,19}$, no puede excluirse antes de evaluar la respuesta al tratamiento con hormona tiroidea. La medición de la subunidad alfa -que es parte de todas las hormonas glicoproteicas (LH, FSH, TSH, HCG)-, puede constituir un examen de gran utilidad cuando se sospecha un tumor hipofisiario productor de $\mathrm{TSH}^{5}{ }^{56}$, ya que sus niveles circulantes aumentan considerablemente en estos casos.

En nuestra paciente la tomografía axial y la resonancia nuclear sugerían macroadenoma hipofisiario. Los exámenes por imágenes, sin embargo, no son capaces de diferenciar entre macroadenoma e hiperplasia hipofisiaria, pues los criterios de diagnóstico radiológico son similares en ambas entidades 5 . Por lo tanto es fundamental el estudio de función tiroidea frente a un paciente con aumento de volumen hipofisiario $5.6,8$.

En el caso que se comenta, la decisión de hacer una prueba terapéutica con hormonas tiroideas se basó en la presencia de hipotiroidismo confurmado clínica y bioquímicamente con hormonas tiroideas bajas y TSH elevado; el hallazgo de una tiroides sublingual en la cintigrafia tiroidea, sospechado clínicamente por la ausencia de tiroides palpable normotópico en el examen físico y la ausencia de síntomas y signos neurológicos que pudieran ser causados por un macroadenoma pituitario.

Concluimos que la prueba terapéutica con hormona tiroidea constituye una herramienta fundamental para el diagnóstico diferencial de la hiperplasia hipofisiaria por hipotiroidismo primario con otros procesos expansivos intraselares. Este tratamiento médico habitualmente causa una regresión total del crecimiento hipofisiario en casos de hipotiroidismo primario, evitando la intervención del neurocirujano $0^{5} .8 .11,12$. El diagnóstico precoz del hipotiroidismo prima- 
rio mediante un acucioso examen clínico? , juega un rol fundamental en la prevención de las consecuencias de este sobre el crecimiento y desarollo.

\section{Referencias}

1. La Frunchi SH: Hypothyroidisı. Pediatr Clin North Ain 1979: 26: 33-5i.

2. Bames ND: Screening for congenital hypothyroidism: The first decade. Arch Dis Child 1985; 60: 587-592.

3. Abodousky N, Viwanco X, Cuello X, Perez P. Programa nacional de hipotiroidisıno congénito. Resultados de 24 meses de observación. Rev Chil Pediatr 1995; 3: 140-144.

4. Costin $G$ : Endocrine disorders with tumors of the pituitary and hypothalamus. Pediatr Clin North Am 1979; 26:15-31.

5. Sarlis NJ, Bracker-Davis F. Doppman $J$. and Skarulis MC: MRI-Demonstrable regression of a pitujary mass in a case of primary hypothyroidism after a week of acute thyroid hormone therapy. J Clin Endocrinol Metab 1997; 82: 808-811.

6. Sitver BJ. Kyner $U$, Dick AR. Chang CHJ: Primary hypothytoidism: suprasellar pituitary enlargement and regresston on computed tomographic scanning. JAMA 1981; 246: 364-365.

7. Atchison JA, Lee PA, Albright AL: Reversible suprasellar pituitary mass secondary to hypothyroidism. JAMIA 1989:262:3175-3177.

8. Valenta $L$, Tamkin $J$, Sostrin $R$, Efíss AN. Eisenberg E: Regression of a pituitary adenoma following levothyroxine therapy of primary hypothyroidism. Fertil Steril 1983; 40: 389-392.

9. Scheithauer BW. Kovacs $K$, Randall $R V$, Ryan $N$ : Pituitary gland in hypothyroidism. Histologic and immunocytologic study. Arch Paihol Lab Med 1985; [09: 499-504.
10. Beck-Peccuz P, Brucker-Davis $R$. Persani $L$. Snatlrridge $R C$, Weintraub $B D$ : Thyrotropin-secreting pituitary tumors. Endocr Rev 1996; 17: 610-638.

11. Adams C, Dean HH. Israeds SI, Patton A, Fewer DH: Primary hypothyroidism with intracranial hypertension and pituitary hyperplasia. Pediatr Neurol 1994; 10: 166-168.

12. Pifa JC Jr. Shajey SH, and Pina R: Diminution of large pituitary tunor after replacement therapy for primary hypothyroidism. Neurology 1979; 29: II691172.

13. Lecky BRF, Lightnun SL, Williams TDM, Plant GT. Stevens J: Myxoedema presenting with chiasmal compression: Resolution after thycoxine replacement. Lancet 1987; 1: 1347-1350.

14. Katz MS. Gregerman Rl, Horvath E. Kovacs $K$, and Ezrin C: Thyrotroph cell adenoma of the human pituitary gland associated with primary hypothyroidism: clinjcal and morphological features. Acta Endocrinol 1980; 95: 41-48.

15. Yamanoto $K$, Saito $K$. Takai $T$. Naito $M$, and Yoshida $S$ : Visual field defects and pituitary enlargement in primary hypothyroidism. J Clin Endocrinol Metab 1983: 57: 283-287.

16. Cesundheit $N$, Petrick PA. Nissim $M$. et at: Thyrotropin-secreting pituitary adenomas: clinical and biochemical heterogeneity. Ann lnt Med 1989; 111: $827-835$.

17. Chan AW, MacFarlane JA. Foy PM. Miles JB: Pituitary enlargement and hyperprolactiuaemia due to primary hypothytoidism: errors and delays in diagnosis. Br J Neurozurg 1990; 4: 107-112.

18. Beck-Peccoz P, Persani L, Asteria C, et al: Thyrotropin secreting pituitary tumors in hyper and hypothyroidism. Acta Med Austríaca 1996: 23; 41 46.

19. Langtois $H F$, Lamarche JB, Bellabarba D: Long standing goiter and hypothyroidism: an unusual presentation of a TSH secreting adenoma. Thyroid $1996 ; 6: 329-335$. 